\title{
The realization of an integrated Mach-Zehnder waveguide immunosensor in silicon technology
}

\author{
E.F. Schipper ${ }^{a, *}$, A.M. Brugman ${ }^{a}$, C. Dominguez ${ }^{b}$, L.M. Lechuga ${ }^{c}$, \\ R.P.H. Kooyman ${ }^{\text {a }}$, J. Greve ${ }^{a}$ \\ a BioInterface Group, Mesa Research Institute, Department of Applied Physics, University of Twente, PO Box 217, \\ 7500 AE Enschede, Netherlands \\ ${ }^{b}$ Centro Nacional de Microelectrónica, Campus UAB, 08193 Bellaterra Barcelona, Spain \\ ${ }^{c}$ Centro Nacional de Microelectrónica, Calle Serrano 144, 28006 Madrid, Spain
}

Received 23 February 1996; received in revised form 12 August 1996; accepted 16 August 1996

\begin{abstract}
We describe the realization of a symmetric integrated channel waveguide Mach-Zehnder sensor which uses the evanescent field to detect small refractive-index changes $\left(\Delta n_{\min } \approx 1 \times 10^{-4}\right)$ near the guiding-layer surface. This guiding layer consists of ridge structures with a height of $3 \mathrm{~nm}$ and a width of $4 \mu \mathrm{m}$ made in $\mathrm{Si}_{3} \mathrm{~N}_{4}$. This layer has a thickness of $100 \mathrm{~nm}$. The sensor device has been tested with glucose solutions of different bulk refractive indices. Results of a slab-model calculation are in good agreement with obtained experimental results. The feasibility of applying this sensor for immunosensing, detecting directly the binding of antigen to an antibody receptor surface, is shown with antibody-antigen binding experiments. (C) 1997 Elsevier Science S.A.
\end{abstract}

Keywords: Immunosensors; Mach-Zehnder principle; Evanescent waves; Channel waveguides

\section{Introduction}

Optical evanescent-wave sensors based on the Mach-Zehnder principle show a very high sensitivity for the detection of refractive-index changes on a waveguide surface within the evanescent field of the waveguide. Due to this high sensitivity, this type of sensor is suitable for direct detection of antigen binding to antibody receptor molecules immobilized on the waveguide surface. With a direct detection measurement the number of preparation steps in an immunoassay is smaller than in indirect detection, and also on-line measurements will become possible.

Heideman et al. [1] have shown that with a slab waveguide Mach-Zehnder sensor, a bulk refractiveindex change of $\approx 4 \times 10^{-6}$ could be detected, which is equivalent to a protein surface coverage of approxi-

\footnotetext{
* Corresponding author. Tel: +31534893157 . Fax: +3153489 1105 .
}

mately $1 \mathrm{pg} \mathrm{mm}^{-2}$. However, a disadvantage of this earlier Mach-Zehnder sensor is its 'bulky' construction, making it more complex in operation than, for example, surface plasmon resonance (SPR)-based evanescent-wave sensors. Also the development of future multichannel sensors is impossible when this concept is used. In this paper we shall demonstrate that with silicon technology more integrated and, therefore, less complex Mach-Zehnder sensors can be made. It will be shown that with $\mathrm{Si}_{3} \mathrm{~N}_{4}$ waveguiding channels, consisting of $3 \mathrm{~nm}$ ridges, immunoreactions can be monitored. Channel waveguide interferometer sensors have earlier been used as gas sensors $[2,3]$. However, those sensors are less suitable as immunosensors. They were made with ion-exchanged channel waveguides which will show less surface sensitivity for detection of immunobinding reactions than $\mathrm{Si}_{3} \mathrm{~N}_{4}$ channel waveguides. The Mach-Zehnder device presented here could be the forerunner of a new generation of immunosensors capable of sensitive multichannel operation. 


\section{Principle}

Here we shall only give a very short description of the mode behaviour in a channel waveguide. For a more detailed discussion we refer to the review by Syms and Cozens [4].

In our device light with a transverse field distribution $E=E(x, y)$ is transported by a channel waveguide, see Fig. 1. This light enters a $Y$ junction where it is split into two parts, $E_{1}(x, y)$ and $E_{2}(x, y)$, both dependent on the field distribution $E(x, y)$ before the splitter:

$$
\begin{aligned}
E_{\mathrm{tot}}(x, y) & =E_{1}(x, y)+E_{2}(x, y) \\
& =a E(x+S / 2, y)+b E(x-S / 2, y)
\end{aligned}
$$

Here $S$ is the distance between the two arms, see Fig. 1. In the case of monomode channels $a$ and $b$ are constants; when there are no losses in the $\mathrm{Y}$ junction then $a^{2}+b^{2}=1$.

When after the $Y$ junction one of the beams interacts with an analyte, a phase shift $\phi$ between both beams will occur. If we have monomode channels we may write in this case for the overall field distribution $E(x, y)$ and the phase difference $\phi$, after the interaction area:

$E_{\text {tol }}(x, y)=a E(x+S / 2, y) \exp \mathrm{j} \phi+b E(x-S / 2, y)$

with

$\phi=(2 \pi / \lambda) \Delta N_{\text {eff }} L$

Here $\hat{\lambda}$ is the wavelength, $\Delta N_{\text {eff }}$ is the effective refractive-index difference between both channels as a result of the analyte interaction, and $L$ is the interaction length (length of the sensor interface).

In the Mach-Zehnder sensor both light beams are recombined in a second $\mathrm{Y}$ junction. When these light beams are out of phase, higher-order modes will be excited. In a monomode channel these higher-order modes cannot exist and the light will be radiated out of the channel. In this case the light intensity of the resulting waveguide mode is related to the phase difference $\phi$ according to:

$I=A+B \cos (\phi)$ with $B \leq A$

The optimal visibility $V=(A-B) /(A+B)$ is found when $B=A$. This is the case when the $\mathrm{Y}$ junctions are completely symmetric $(a=b)$.

\section{Sensitivity as an immunosensor}

In a waveguide Mach-Zehnder interferometer a sensor area can be made by partly removing the cladding on the waveguide layer surface. Then this device is capable of sensing refractive-index changes at this surface within the evanescent-field volume of the waveguide mode (typically within a few hundreds of nanometres from the surface). These refractive-index changes can be caused by the physical adsorption of proteins to the surface $[1,5,6]$, or for example immunobinding reactions of antigen to antibodies, where the antibodies or the antigen are the receptor molecules that have been immobilized on the surface $[1,5,6]$.

A slab waveguide can be described as a channel waveguide of which the effective refractive index in the channel $\left(N_{\text {eff }}\right)$ is the same as that in the surroundings of the channel $\left(N_{\text {efr }}\right)$, see Fig. 1. In our design the effective refractive-index difference between channel and surroundings is kept low. For this reason we expect that we can use a slab-waveguide model to predict the properties of the waveguides used in the present paper. The dependence of the effective refractive index $\left(N_{\text {eff }}\right)$ on variations in the thickness $t_{\mathrm{f}}$ of an adsorbing layer has been previously calculated $[7,8]$ in such a slab waveguide model. With the help of Eqs. (2), (3) and (4), the differential sensitivity can now be calculated:

$\frac{\mathrm{d} I}{\mathrm{~d} t_{\mathrm{f}}}=\frac{\mathrm{d} I}{\mathrm{~d} \phi} \frac{\mathrm{d} \phi}{\mathrm{d} \Delta N_{\text {eff }}} \frac{\mathrm{d} \Delta N_{\text {eff }}}{\mathrm{d} t_{\mathrm{f}}}$

In immunosensing experiments the average thickness of the layer grown on the surface as a result of the immunobinding reaction is related to the fraction of occupied receptor sites $(\Gamma)$, which is dependent on the concentration $(C)$ of the analyte molecules in the sample solution [4], on the density of receptor molecules on the surface $(\alpha)$, and on the molecular weight $(M)$ of the analyte molecules [5]:

$t_{\mathrm{f}}=f(\Gamma(C), \alpha, M)$

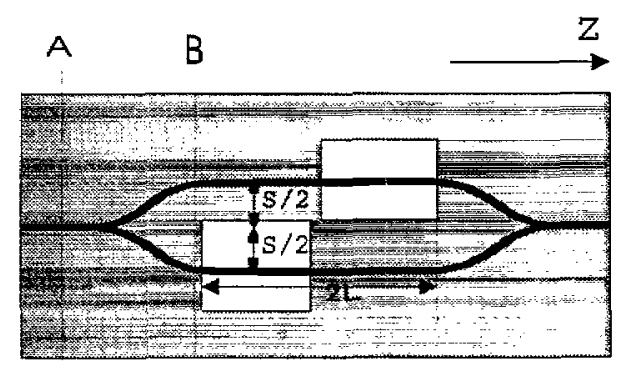

(a)

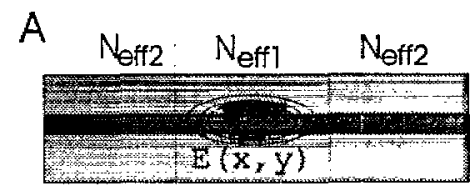

B

(b)
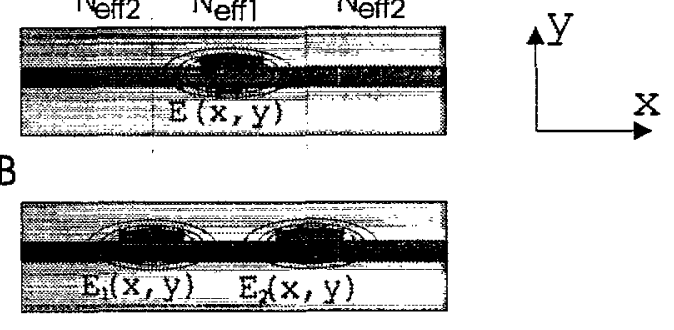

Fig. 1. Channel waveguide Mach-Zehnder sensor: (a) top view; (b) cross sections at A and B, respectively. For explanation of symbols, see text. In (b) the electric-field profile is indicated. 


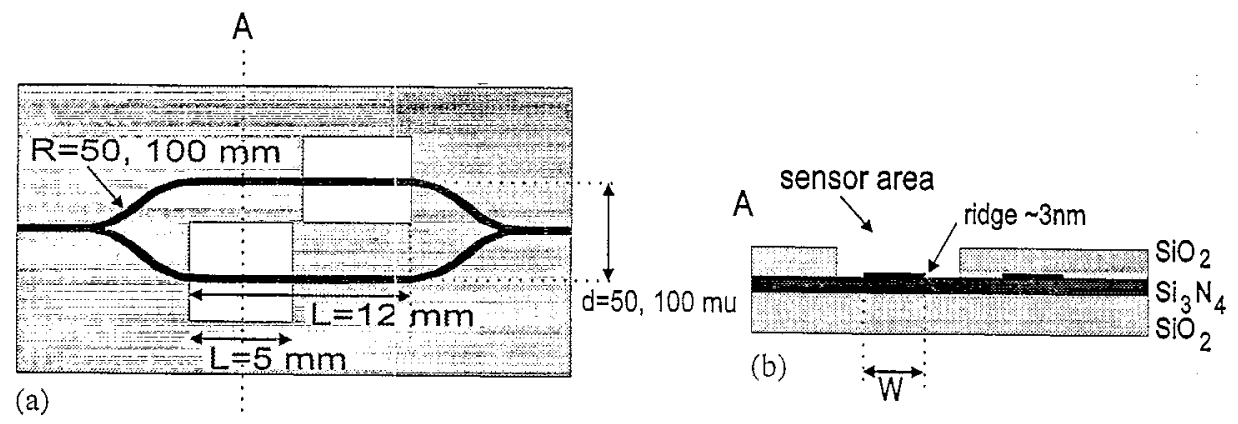

Fig. 2. Design of the integrated Mach-Zehnder sensor, for details see text: (a) top view; (b) cross section at A.

\section{Device structure and manufacture}

The design of the Mach-Zehnder device can be seen in Fig. 2. It has been realized in Si technology for operation with a $632.8 \mathrm{~nm}$ HeNe laser. A $1.5 \mathrm{~mm}$ thermally oxidized $\mathrm{SiO}_{2}$ layer with a refractive index of 1.46 is used as the lower cladding layer. On this $\mathrm{SiO}_{2}$ layer a high-index LPCVD $\mathrm{Si}_{3} \mathrm{~N}_{4}$ waveguide layer with a refractive index of 2.0 , and a $1.5 \mu \mathrm{m}$ PECVD $\mathrm{SiO}_{2}$ cladding layer with a refractive index of 1.46 are deposited. The high refractive-index contrast between waveguide layer and cladding is chosen to obtain a maximum sensitivity $[7,8]$. This is especially needed for immunosensing experiments where the average thickness of the layer grown on the surface will be smaller than $1 \mathrm{~nm}$ [5]. For a TE0 mode in a slab waveguide the highest sensitivity is found at a waveguide layer thickness of around $100 \mathrm{~nm}$ [1]. Three batches of channel waveguides were manufactured, with wavegguide layer thickness of 100 (I), 200 (II) and 400 (III) nm, respectively.

The realization of channel waveguides in this structure is not straightforward, in view of the very small ridge height of a few nanometres required for monomode operation [8]. With reactive ion etching (RIE) technology it turned out to be possible realize a ridge hight of $\approx 3 \pm 1 \mathrm{~nm}$ in $\mathrm{Si}_{3} \mathrm{~N}_{4}$, as measured by atomic force microscopy. The error is completely determined by the roughness of $\mathrm{Si}_{3} \mathrm{~N}_{4}$. In this way semimonomode waveguide channels, which are monomode in the $y$ direction but still bimodal in the $x$ direction could be realized. These channel waveguides have a width $(W)$ of $4.0 \pm 0.5 \mu \mathrm{m}$ in the $x$ direction.

The $Y$ junction splitter and combiner are designed with S-bend structures. As noted by Syms [4], for a reasonable light confinement within channels the bend radius of an S-bend structure should be more than 10 $\mathrm{mm}$. In our design radii $(R)$ of 50 or $100 \mathrm{~mm}$ were used. With these bend radii no extra light losses due to the bending were observed. The total length of the $Y$ junction splitter and combiner is kept at $30 \mathrm{~mm}$. With this length the light is split into two branches that are 50 or $100 \mathrm{~mm}$ separated from each another. No differences in operation of these two devices were observed. For this reason they were used interchangeably.

On both branches of the interferometer a sensor area is created with a length $(L)$ of $5 \mathrm{~mm}$. This is done by removing the $\mathrm{SiO}_{2}$ cladding in an $\mathrm{HF}$ etching step. Due to the fact that the $\mathrm{HF}$ etching rate of $\mathrm{SiO}_{2}$ is 200 times faster than that of $\mathrm{Si}_{3} \mathrm{~N}_{4}$, a good sensor window can be made in this way. A sensor area is created on both branches of the interferometric device to keep the optical differences between the branches as small as possible. In this way temperature drifts that normally limit the performance of a Mach-Zehnder sensor are kept small.

\section{Experimental}

The set-up consists of a $2.5 \mathrm{~mW} 632.8 \mathrm{~nm}$ polarized $\mathrm{HeNe}$ laser, an end-fire in- and outcoupling construction with two $40 \times, \mathrm{NA}=0.65$ microscope objectives, a photodiode and a sensor chip made in silicon, as shown in Fig. 3. Light is coupled into the sensor chip with the first microscope objective by focusing the laser beam perpendicularly onto the polished end-face of the device. The incoupled light travels through the device and is out-coupled and projected onto the photodiode. This photodiode is shielded for scattered light with a

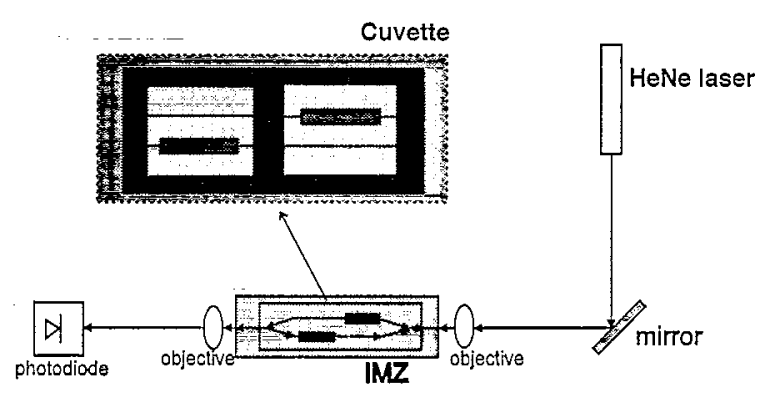

Fig. 3. The Mach-Zehnder set-up with cuvette. 
diaphragm. On top of the sensor chip a delrin cuvette can be positioned. The sensor areas on the chip are designed in such a way that it is possible to position the cuvette without the help of a dedicated micropositioning tool (see Fig. 3). After this it is possible to apply the analyte sample to one of the sensor areas keeping a reference sample (without analyte molecules) on the other sensor area during a measurement.

To calibrate the sensor chip a number of solutions were prepared with different glucose concentrations, with refractive indices in the range $(1.3358-1.3451) \pm$ $2 \times 10^{-4}$, as determined by an Abbe refractometer, operating at $t=20^{\circ} \mathrm{C}$.

Immunosensing experiments were performed with anti human chorionic gonadotropin (anti-hCG, mol. $\mathrm{wt} .=150 \mathrm{kD}$ and human chorionic gonadotropin (hCG, mol. wt. $\approx 42 \mathrm{kD}$ ) provided by Organon International (Netherlands), and bovine serum albumin (BSA, mol. wt. $\approx 65 \mathrm{kD}$ ) bought from Sigma Chemical Co. (USA). All immunosensing experiments were done in phosphate buffered saline (PBS) with a $\mathrm{pH}$ of 7.35 at room temperature $\left(20^{\circ} \mathrm{C}\right)$.

\section{Results and discussion}

\subsection{Model verification}

The Mach-Zehnder sensor as an immunosensor detects the binding of analyte molecules to receptor molecules immobilized on the sensor surface. The effect of binding is comparable to a change of the bulk refractive index of the analyte medium itself [3]. Therefore, to verify the applicability of model calculations to the sensor response, the use of solutions with different bulk refractive indices is practical. To this end, solutions with different concentrations of glucose in water were used. The response of the Mach-Zehnder sensor to these solutions is measured and compared with the model expectations.

Batches I-III were analysed for TE-mode operation: as already mentioned, batch I corresponds to an expected maximum sensitivity; batch II is still monomode in the $y$ direction, and batch III has a lossy confinement of the higher-order mode in the $y$ direction.

First, the response of these sensors to a bulk refractive-index step of $9.5 \times 10^{-3}$ was determined. The results of these measurements can be found in Table 1 . As expected, the biggest response is measured with batch I. For batch II a three times lower response is measured, and for batch III even a 20 times lower response is found. As we can see in the Table, the measured values are in reasonable agreement with the slab-guide model calculations. The visibility of these measured interference signals varied between 0.50 and 0.01 for the various batches; it appeared to be corre-
Table 1

Sensitivity of three different Mach-Zehnder sensors for a bulk refractive-index step of $9.5 \times 10^{-3}$

\begin{tabular}{llll}
\hline Batch & $\begin{array}{l}\text { Waveguide layer } \\
\text { thickness } \\
(\mathrm{nm})\end{array}$ & $\begin{array}{l}\text { Calculated } \\
\text { phase change } \\
(\times 2 \pi)\end{array}$ & $\begin{array}{l}\text { Measured } \\
\text { phase change } \\
(\times 2 \pi)\end{array}$ \\
\hline I & 100 & $12.1 \pm 0.3$ & $14.0 \pm 0.3$ \\
II & 200 & $4.5 \pm 0.1$ & $4.5 \pm 0.3$ \\
III & 400 & $1.1 \pm 0.02$ & $0.5 \pm 0.5$ \\
\hline
\end{tabular}

lated to the quality of the $Y$ junctions of the devices, dependent on the lithography during manufacture. Subsequently the response of batch I was subjected to a more severe test by measuring the phase change as a function of the refractive index, using the glucose solutions. The results of these measurements are depicted in Fig. 4. The line in this graph gives the theoretical response following from the slab-guide model. As can be seen, the measured phase change is in all situations comparable to the model calculations. This shows again that our devices work as expected and that a 'simple' slab-waveguide model can be used to calculate the sensitivity. These measurements also provide a calibration for the device that was used for immunoexperiments (see next section). The differential sensitivity $\mathrm{d} \phi / \mathrm{d} n$ of this device, calculated from a least-square fit on the experimental data, was found to be $(1.4 \pm$ $0.1) 10^{3} \times 2 \pi$. The two-fold higher sensitivity previously found in the planar Mach-Zehnder device [1] can be fully accounted for by the longer interaction length in this device. From Fig. 4 it can also be inferred that it is possible to measure a bulk refractive-index change of $2 \times 10^{-3}$. From calculations presented earlier [1] we can conclude from this that it is easily possible to detect an average protein layer growth of $1 \mathrm{~nm}$.

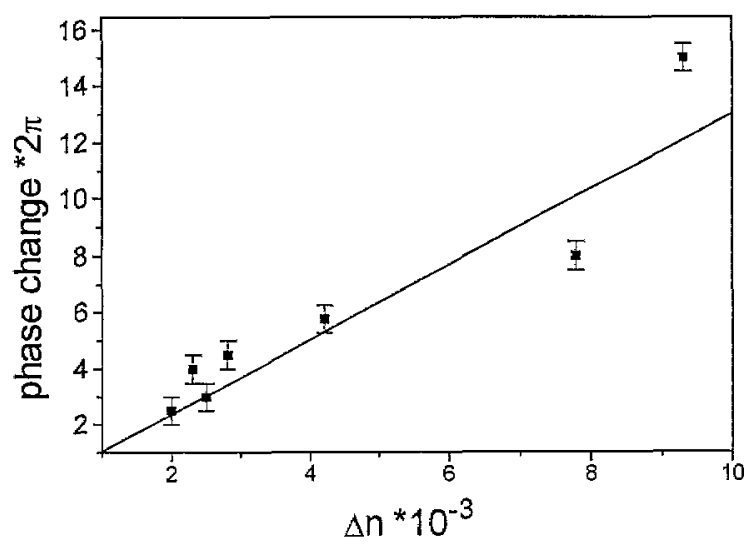

Fig. 4. Response of the Mach-Zehnder sensor as a function of bulk refractive index. The waveguide layer thickness is $100 \mathrm{~nm}$. 


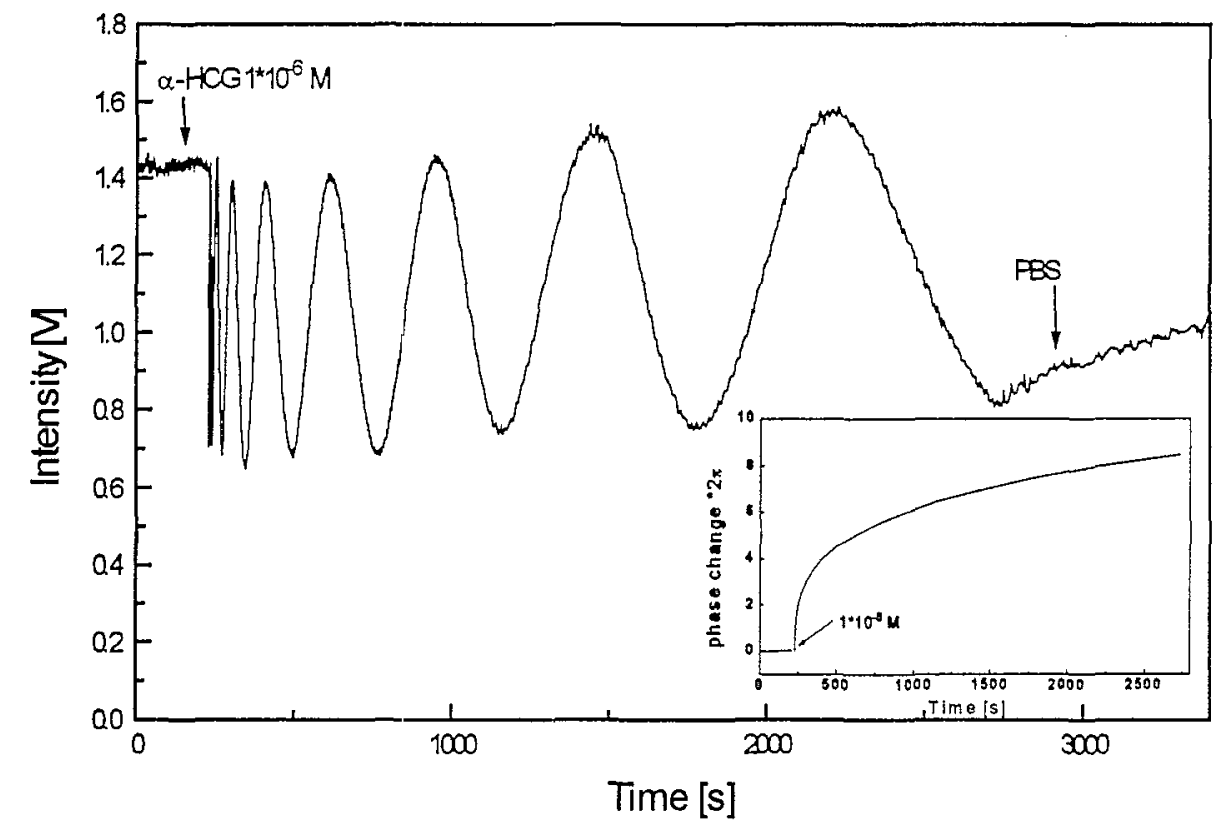

Fig. 5. Physical adsorption of $1 \times 10^{-6} \mathrm{M}$ anti-hCG antibodies on the sensor surface as a function of time. The inset shows the response if we measure the number of fringes that pass during the adsorption process.

\subsection{Immunosensing experiments}

According to Heideman et al. [1] the binding of hCG to an anti-hCG receptor surface, prepared by physical adsorption, results in an average layer growth of at most $\approx 0.3 \mathrm{~nm}$. According to our calibration experiments, this results in a phase change $\approx 2 \pi$. This phase change should be detectable.

The preparation of the receptor surface by physical adsorption of $1 \times 10^{-6} \mathrm{M}$ anti-hCG to a plasma- $0_{2^{-}}$ cleaned surface is measured and can be seen in Fig. 5 . Before application of the antibody solution the phase drift of the sensor is $<0.5 \pi \mathrm{h}^{-1}$. This is probably due to the combined effect of temperature drift $\left(<0.5^{\circ} \mathrm{C}\right)$ and instability of the receptor surface. As can be seen in Fig. 5, the effect of an adsorption process in the same time span is much more pronounced. The complete phase change after a $2 \mathrm{~h}$ adsorption is $\approx 10 \times 2 \pi$. The slight increase in average intensity level during the adsorption is ascribed to increased roughness as a result of protein adsorption [9]. As expected, the phase change is fastest in the first period of the process. It can also be seen that after a PBS washing step the phase drift again becomes $<0.5 \pi \mathrm{h}^{-1}$, comparable with the initial phase drift before the adsorption process.

Before the adsorption experiments were done it was not sure if after a coating process the channels would still work properly. The ridge thickness of these channels is $\approx 3 \mathrm{~nm}$. Adsorbing antibodies have the same dimensions, and thus they could increase the roughness of the surface to such a level that the channels disappear in the roughness.
Fortunately this did not take place. It was only found that in the beginning of the adsorption process, when the intensity modulation is most prominent, the visibility is somewhat smaller, possibly as a result of increased surface roughness due to the adsorption process.

After the preparation of the anti-hCG receptor surface the specificity of this surface for the immunosensing of hCG is tested. This is done by adding a concentration of BSA to the surface. Adding $1 \times 10^{-6}$ $\mathrm{M} \overline{\mathrm{B}} \overline{\mathrm{SA}}$ gives no response. Affter this specificity test, hCG is added at the same temperature in concentrations ranging from $10^{-8}$ to $10^{-7} \mathrm{M}$. In Fig. 6 we see

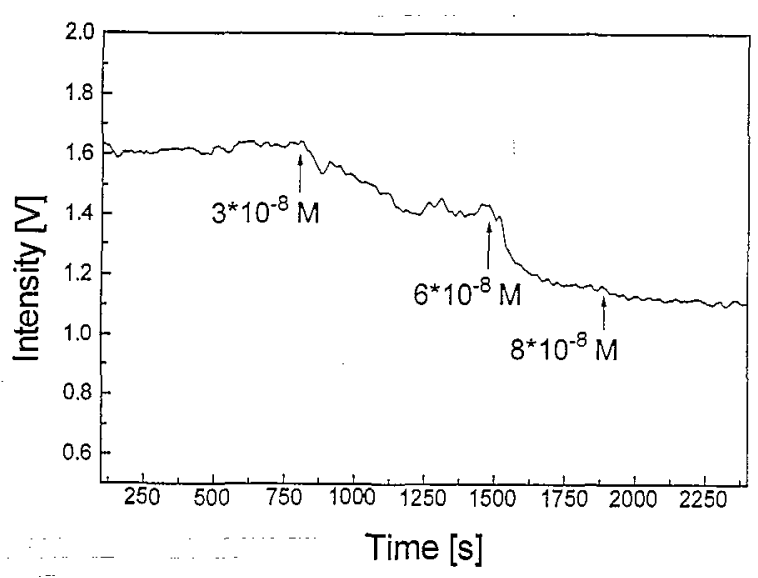

Fig. 6. Immunosensing experiment with the integrated Mach-Zehnder sensor. The sensor surface is coated with anti-hCG. hCG is added at the indicated instants at concentrations shown below the arrows. 
the familiar binding curves, well known from previous reports. Both the addition of $3 \times 10^{-8} \mathrm{M}$ and, subsequently, $6 \times 10^{-8} \mathrm{M}$ result in an immunoresponse. Addition of $6 \times 10^{-8} \mathrm{M}$ antigen almost saturates the receptor layer, as is seen by the subsequent addition of $8 \times 10^{-8} \mathrm{M}$ of antigen, where almost no response is found any more. This behaviour is completely in line with earlier results [1].

These experiments show that it is feasible to use the integrated Mach-Zehnder sensor as an immunosensor for the direct detection of the binding of antigen to antibodies on the sensor surface.

\section{Conclusions}

We have demonstrated that it is possible to manufacture sensitive monomode channel waveguides by etching a small ridge of $\approx 3 \mathrm{~nm}$ in $\mathrm{Si}_{3} \mathrm{~N}_{4}$ waveguide layers. These waveguide layers have an optimized thickness of $100 \mathrm{~nm}$. Also after adsorption of antibodies on these channel waveguides the mode character in the channels remains unchanged, which makes them attractive for immunosensor applications.

For glucose solutions with different refractive indices it has been shown that the Mach-Zehnder devices work as anticipated and that the sensitivity of these devices can be calculated with a simple slab-waveguide model. The feasibility of these devices as immunosensors for the direct detection of antigen binding to an antibody receptor surface has been shown with hCG/ anti-hCG measurements.

The compact and simple design of the sensor opens possibilities for future development of multichannel devices, which are difficult to realize with slab-waveguide-based Mach-Zehnder sensors.

For a further improvement of the sensor system two main aspects deserve further attention: (1) If it is possible to construct narrower channels with a uniform width, the resulting optical behaviour will become really monomodal. Consequently the visibility will be much improved, resulting in a more sensitive response. (2) For the direct detection of the immunobinding of low concentrations of antigen to an antibody receptor surface, where the expected phase change is much smaller than $2 \pi$, fringe counting, which is appropriate for the detection of adsorption processes, is not accurate enough. A measurement of a phase change smaller than $2 \pi$ based on direct measurement of the change in the intensity of the outcoupled light beam is also highly non-linear and dependent on the initial phase difference between reference and signal beam. Only a phase-tuning system can adequately address these shortcomings of the current fringe-counting method. Work on these points is in progress.

\section{Acknowledgements}

These investigations have been supported by the Dutch Technology Foundation (STW).

\section{References}

[1] R.G. Heideman, R.P.H. Kooyman and J. Greve, Performance of a highly sensitive optical waveguide Mach-Zehnder interferometer immunosensor, Sensors and Actuators B, 10 (1993) 209-217.

[2] N. Fabricius, G. Gauglitz and J. Ingenhoff, A gas sensor based on an integrated optical Mach-Zehnder interferometer, Sensors and Actuators B, 7 (1992) 672-676.

[3] A. Brandenberg, R. Edelhäuser and F. Hutter, Integrated optical gas sensors using organically modified silicates as sensitive films, Sensors and Actuators B, 11 (1993) $361-374$.

[4] R. Syms and J. Cozens, Optical Guided Waves and Devices, McGraw-Hill, London, 1992.

[5] E.F. Schipper, R.P.H. Kooyman, R.G. Heideman and J. Greve, Feasibility of optical waveguide immunosensors for pesticide detection: physical aspects, Sensors and Actuators B, 24-25 (1995) 90-93.

[6] E.F. Schipper, R.P.H. Kooyman, A. Borreman and J. Greve, The critical sensor: a new type of evanescent wave immunosensor, Biosensors Bioelectron., 11 (1996) 295-304.

[7] K. Tiefenthaler and W. Lukosz, Sensitivity of grating couplers as integrated-optical sensors, J. Opt. Soc. Am. B, 6(1989) 209-220.

[8] W. Lukosz, Principles and sensitivities of integrated optical and surface plasmon sensors for direct affinity sensing and immunosensing, Biosensors Bioelectron., 6 (1991) 215-225.

[9] A.N. Sloper and M.T. Flanagan, Scattering in planar surface waveguide immunosensors, Sensors and Actuators B, 11 (1993) $537-542$.

\section{Biographies}

Eddy Schipper was born in Brunssum, The Netherlands, in February 1968. He received his M.S. degree in applied physics in August 1991. From 1991 to 1993 he worked on the development of an optical absorption sensor to obtain a degree in design in modern optics in 1993. He is presently working towards his $\mathrm{Ph} . \mathrm{D}$. degree by developing waveguide sensors for the detection of small molecules at the Biointerfaces group.

Antoinette Bnigman was born in Ede, The Netherlands, in November 1970. She worked at the Biointerfaces group to obtain her M.S. degree in applied physics in August 1995.

Carlos Dominguez received the B.S., M.S. and Ph.D. degrees in chemistry from the Universidad Complutense of Madrid, Spain, in 1980, 1981 and 1985, respectively. He became a member of the scientific staff at the Instituto de Microelectrónica de Barcelona (IMB-CNM, CSIC) in 1986. Since 1991 he has been a senior scientific researcher at IMB-CNM, CSIC. He is involved in chemical vapour deposition processes and 
wet and dry etch processes. Currently he is working on the development of an integrated optical technology based on silicon for sensors and broad-band telecommunications applications.

Laura M. Lechuga is a research associate in the National Center for Miroelectronics at the Spanish Council for Research. She is involved in projects about the development of sensors and biosensors based on III-V semiconductor technology. Earlier, she has worked at the Biointerfaces group at the Lniversity of Twente, The Netherlands. Her background is chemistry (University of Cádiz, Spain) and III-V serniconductor devices (University Complutense of Madric, Spain).

Rob Kooyman received his M.S. degree in physics at Leiden University in 1975. From 1975 to 1980 he worked at the Agricultural University of Wageningen on a Ph.D. thesis devoted to molecular spectroscopy.
At the University of Utrecht he developed spectroscopic methods for use in membrane biophysics. Since 1985 he has been involved in biointerface research at the University of Twente.

Jan Greve was born in Koog aan de Zaan, The Netherlands, in October 1939. He received his M.S. degree in experimental physics from the Free University, Amsterdam, The Netherlands, in 1967 and the Ph.D. degree from the same University in 1972. Since 1967 he has been working in the field of biophysics, applying and developing optical and spectroscopic techniques for the research of biological macromolecules, bacteriophages and cells. In 1980 he started a Biophysics subgroup in the Applied Physics Department of the University of Twente. The current research interests of the group are biointerfaces, biomedical optics, single molecule and cell analysis, and microspectroscopy. 